\title{
Comparison of Land-Mark-Based and Curve-Based Thin-Plate Warps for Analysis of Left-Ventricular Motion from Tagged MRI
}

\author{
A. A. Amini, Y. Chen, and D. Abendschein \\ CVIA Laboratory \\ Washington University Medical Center \\ Campus Box 8086, 660 S. Euclid Ave., St. Louis, MO 63110, USA \\ amini@cauchy. wustl. edu \\ http://www-cv. wustl.edu/
}

\begin{abstract}
MRI is unique in its ability to non-invasively and selectively alter tissue magnetization, and create tagged patterns within a deforming body such as the heart muscle. The resulting grid patterns define noninvasive tissue markers, providing a mechanism for in-vivo measurement of tissue motion and strain. In this paper, we report on objective comparison of thin-plate spline warps in reconstructing true deformations when using homologous land-mark points and when using homologous curves from tagged MR-image sequences of a cardiac motion simulator. In addition, the number of corresponding land-mark points as well as the number of corresponding curves are varied in order to determine the effect on accuracy of reconstructions. Finally, application of the developed techniques to computing LV tissue motion and strain in systole from short-axis tagged images of a porcine model with anterior myocardial infarction (MI) are given.
\end{abstract}

\section{Introduction}

Non-invasive techniques for assessing the dynamic behavior of the human heart are invaluable in the diagnosis of heart disease, as abnormalities in the myocardial motion sensitively reflect deficits in blood perfusion $[9,17]$. MRI is a non-invasive imaging technique that provides superb anatomic information with excellent spatial resolution and soft tissue contrast. In MR tagging, the magnetization property of selective material points in the myocardium are altered in order to create tag grid patterns within a deforming body such as the heart muscle $[17,7]$. During tissue contractions, the grid patterns move, allowing for visual tracking of the land-mark grid intersections over time. The intrinsic high spatial and temporal resolutions of such myocardial analysis schemes provide unsurpassed information about local contraction and deformation in the heart wall which can be used to derive local strain and deformation indices from different myocardial regions.

A number of researchers have developed different approaches to analysis of tagged MRI data $[16,11,10]$. An unanswered question is whether more accuracy 
is gained in utilizing complete curve-grid information in reconstructing LV deformations, or whether use of tag intersections (i.e., landmarks) alone is sufficient. In this paper, we report on comparisons of land-mark-based and curve-based warps, and describe conditions under which thin-plate reconstructions using complete tag grid information would be preferred. We also apply the reconstruction algorithm to obtain dense strain maps from tagged MRI in a porcine model with anterior MI.

\section{Constrained Thin-Plate Spline Warps}

Tracking tissue deformations with SPAMM using snake grids provides 2D displacement information at tag intersections and 1D displacement information along other 1D snake points [1,2,3,4]. The displacement measurement from tag lines however are sparse; interpolation is required to reconstruct a dense displacement field from which strain, torsion, and other mechanical indices of function can be computed at all myocardial points.

To proceed more formally, the vector field which warps the deformed image into the undeformed image is assumed to be $C^{1}$ continuous. This smoothness requirement is achieved by minimizing an objective function which combines spatial derivatives of both components of the reconstructed displacements

$$
\Phi_{1}=\iint u_{x x}^{2}+2 u_{x y}^{2}+u_{y y}^{2} d x d y+\iint v_{x x}^{2}+2 v_{x y}^{2}+v_{y y}^{2} d x d y
$$

characterizing approximating thin-plate splines. It should be noted that although thin-plate warps have been used in the past for other medical imaging applications ([8]), they have only been utilized to interpolate a warp given homologous landmark points. Assuming 2D tissue motion (as is roughly the case towards the apical end of the heart [13]), two possible physical constraints (in addition to (1)) can be considered:

1. Corresponding homologous land-marks obtained from intersecting horizontal and vertical tag grid lines should come into correspondence after application of the thin-plate warp. The intersection of two tag grid lines are "pulled" towards one another by minimizing

$$
\Phi_{2}=\sum\left(u-u_{i n t}\right)^{2}+\left(v-v_{i n t}\right)^{2}
$$

where $u_{\text {int }}$ and $v_{\text {int }}$ are the $x$ and $y$ components of displacement at tag intersections.

2. Corresponding homologous curves should come into correspondence, that is, any point on a deformed tag in frame $n$ must be warped to lie on its corresponding undeformed tag in frame 0 of the sequence. For a vector field to perform such a warp, $h(u, v)$ of figure 1 must be minimized. Let $\boldsymbol{P}_{1}=$ $\boldsymbol{P}_{1}(u, v)=(x, y)+(u, v)$ as in figure 1 , and let $\boldsymbol{P}_{2}$ be any point on the 
undeformed tag line. The following term is then summed over all deformed horizontal and vertical grid points:

$$
\Phi_{3}=\sum(h(u, v))^{2}=\sum\left\{\left(\boldsymbol{P}_{1}(u, v)-\boldsymbol{P}_{2}\right) \cdot \boldsymbol{n}\right\}^{2}
$$

where the summation is performed on all points $(x, y)$ on the deformed grids.
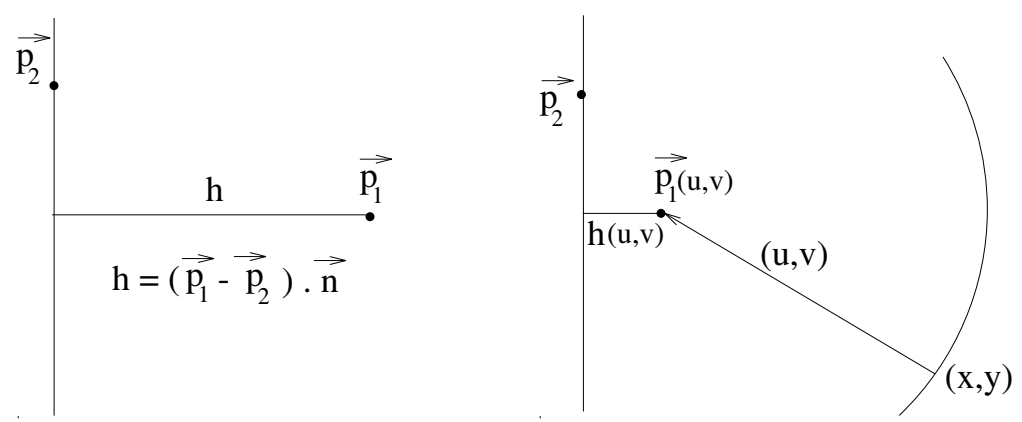

Fig. 1. Distance of a point $P_{1}$ to a straight line can easily be calculated with knowledge of any point, $P_{2}$, on the line and the normal vector (left). The residual distance from a warped point of a deformed tag line to its corresponding undeformed tag line in frame 0 of the sequence, $h(u, v)$, can similarly be calculated (right).

In order to compare curve-warps with land-mark warps, an objective function maybe formed by a linear combination of the terms in (1), (2), and (3),

$$
\Phi=\lambda_{1} \Phi_{1}+\lambda_{2} \Phi_{2}+\lambda_{3} \Phi_{3}
$$

where $\lambda_{1}, \lambda_{2}, \lambda_{3}$ are non-negative weights. The following test cases can then considered: I. $\left\{\lambda_{1}>0, \lambda_{2}>0, \lambda_{3}=0\right\}$, and II. $\left\{\lambda_{1}>0, \lambda_{2}=0, \lambda_{3}>0\right\}$. Additionally, within each of case I (land-mark-based warps) and II (curve-based warps), the number of homologous land-mark points/curves may be varied to determine the effect on accuracy of reconstructions. In order to optimize $\Phi$ with Conjugate-Gradient Descent, partial derivatives of $\Phi_{1}$ can be calculated using the computational molecule approach discussed in [14] and also used in [4]. Partial derivatives of (2) can easily be obtained. Finding partial derivatives of $\Phi_{3}$ is also straightforward:

$$
\nabla \Phi_{3}=2 \sum\left\{\left(\boldsymbol{P}_{1}(u, v)-\boldsymbol{P}_{2}\right) \cdot \boldsymbol{n}\right\} \cdot \boldsymbol{n}
$$

As maybe concluded from the last paragraph, minimization of (4) leads to a system of linear equations with full rank under non-trivial deformations. Therefore, (4) is a convex objective function, with a global minimum. It should be 
noted that in comparison to [4], in the present article we describe thin-plate techniques which require knowledge of homologous landmark points and/or curves; though with an additional constraint, namely that one set of the homologous curves should be straight. This is a modification of the framework in [4], making the objective function convex, bypassing local minima, and resulting in reduced computational costs.

\section{Land-Mark-Based vs. Curve-Based Warps}

An environment based on a 13 parameter kinematic model of Arts et al. [6] has been implemented as was described in [15] for simulating a time sequence of tagged MR images at arbitrary orientation. Based on user-selected discretization of the space between 2 concentric shells and by varying the canonical parameters of the model, both a sequence of tagged MR images as well as a "ground truth" vector field of actual material point deformations are available [4]. The parameters of the motion model, referred to as k-parameters, and the transformations to which they correspond are: $k_{1}$ : Radially dependent compression, $k_{2}$ : Left ventricular torsion, $k_{3}$ : Ellipticalization in long-axis (LA) planes, $k_{4}$ : Ellipticalization in short-axis $(\mathrm{SA})$ planes, $k_{5}$ : Shear in $\mathrm{x}$ direction, $k_{6}$ : Shear in y direction, $k_{7}$ : Shear in $\mathrm{z}$ direction, $k_{8}$ : Rotation about $\mathrm{x}$-axis, $k_{9}$ : Rotation about $\mathrm{y}$-axis, $k_{10}$ : Rotation about z-axis, $k_{11}$ : Translation in x direction, $k_{12}$ : Translation in y direction, and $k_{13}$ : Translation in $\mathrm{z}$ direction. For the purposes of comparing $2 \mathrm{D}$ displacement field reconstructions, we have used the parameters $k_{2}, k_{4}, k_{5}$, and $k_{10}$ for generating $2 \mathrm{D}$ deformations of the geometric model, based on which simulated tagged images and 2D displacement vector fields of actual material points are produced. The error norms used in comparing the ground truth vector field $\left(V_{g}\right)$ with the vector field measured by the warp algorithms $\left(V_{m}\right)$ are: $\varepsilon_{r m s}=\sqrt{\frac{1}{N} \sum\left|V_{m}-V_{g}\right|^{2}}$ and $\varepsilon_{\text {dist }}=\sqrt{\frac{1}{N} \sum(h(u, v))^{2}}$ where $N$ is the total number of deformed grid points on the myocardial region, the summation in $\varepsilon_{r m s}$ is performed on entire myocardial regions, and the summation in $\varepsilon_{\text {dist }}$ is performed on all points $(x, y)$ on the deformed grid (figure 1 ).

In order to test the accuracy of reconstructions as a function of the number of homologous landmarks and homologous curves, a number of experiments were carried out. The experiments were designed to determine the effect of number of homologous land-mark points vs. number of homologous curves on the accuracy of thin-plate spline reconstruction of deformations. The cardiac simulator was used to generate a sequence of SA tagged images in addition to ground-truth displacement vector fields. The imaging parameters chosen resulted in $7 \times 7$ image tag grids. The intersections of the tag lines in the myocardial area yielded all of the available land-mark points, and the tag segments which were in the myocardial region yielded all of the available curves for warping. In order to vary the number of homologous land-marks and curves, the $7 \times 7$ grid was subsampled to a $1 \times 1,2 \times 2,3 \times 3,4 \times 4$, and the original $7 \times 7$ grid. To compare landmark based and curve-based warps, for each subsampled grid considered, the intersections of the corresponding grids were used as the land-mark points. The 

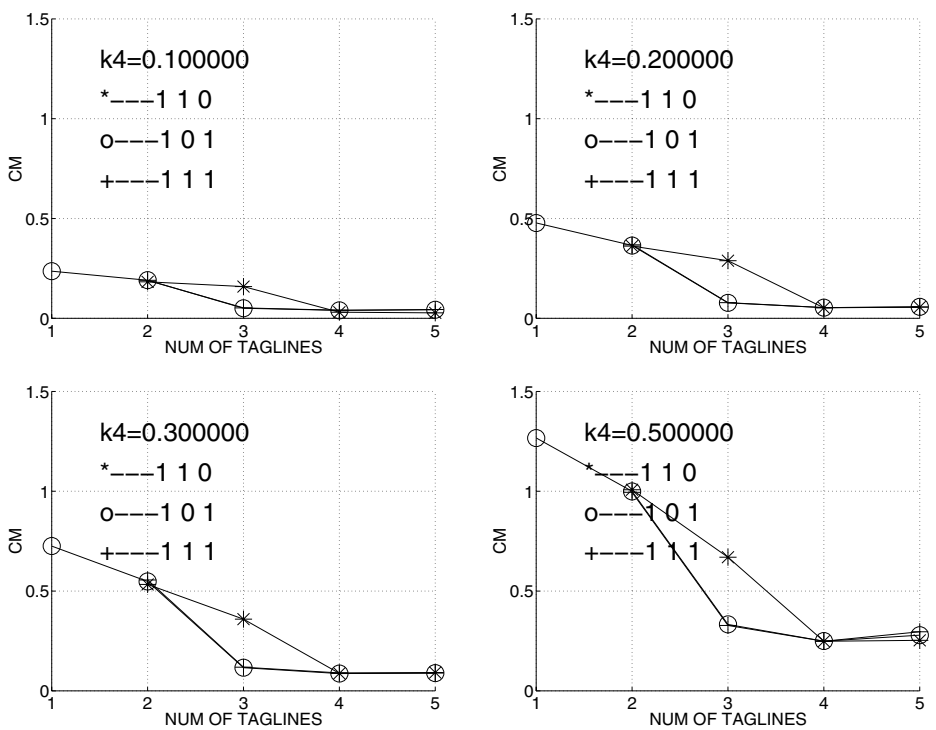

Fig. 2. Plots of $\varepsilon_{r m s}$ as a function of number of tag lines used in thin-plate spline reconstruction of deformations. Different plots correspond to different values of $k_{4}$, ellipticalization in short-axis plane.
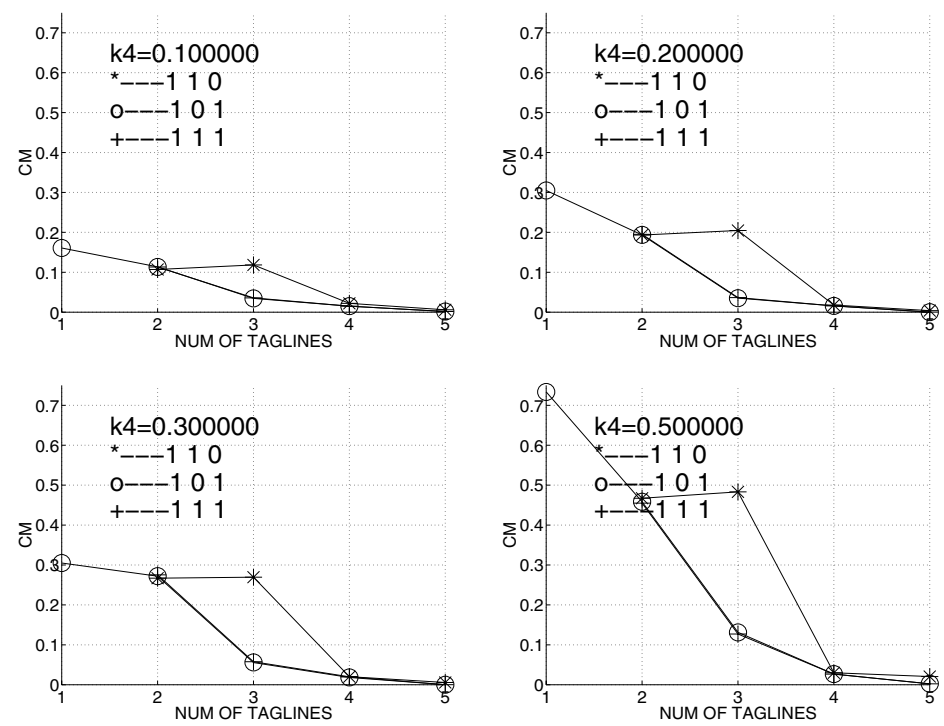

Fig. 3. Plots of $\varepsilon_{\text {dist }}$ as a function of number of tag lines used in thin-plate spline reconstruction of deformations. Different plots correspond to different values of $k_{4}$, ellipticalization in short-axis plane. 
findings for parameter $k_{4}$ (short-axis ellipticalization) are displayed in figures 2 and 3 (plots for $k_{2}, k_{5}$, and $k_{10}$ generally follow the same patterns). Please note that for these figures, there are no land-marks for the $1 \times 1$ grid, and additionally the results of the $7 \times 7$ grid are labeled as case " 5 " on the $\mathrm{x}$-axis of the plots. For the plots, "1 10 " corresponds to $\lambda_{1}=1, \lambda_{2}=1, \lambda_{3}=0$. Similarly, "1 01 " corresponds to $\lambda_{1}=1, \lambda_{2}=0, \lambda_{3}=1$, and "1 11 " corresponds to $\lambda_{1}=1$, $\lambda_{2}=1, \lambda_{3}=1$.

The plots indicate that for small deformations, there is no significant difference between using curve-based, and land-mark based warps, but that for larger deformations, there is more graceful degradation of curve-based warps in reconstruction of true deformations in comparison to land-mark based warps. There are also no significant differences between curve-based and land-mark based warps when the number of land-marks/curves increase. In the rest of the article, $\left\{\lambda_{1}=1, \lambda_{2}=0, \lambda_{3}=1\right\}$ was used for analysis of the in-vivo data.
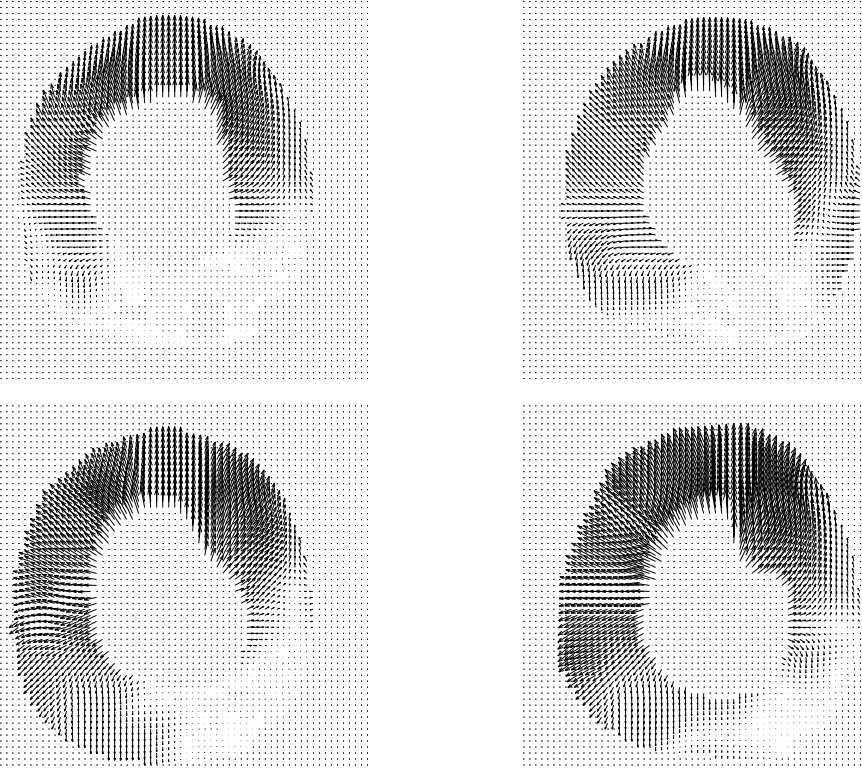

Fig. 4. Four displacement vector fields for the post-MI study corresponding to 202, 225, 247, and $270 \mathrm{msec}$ after the ECG trigger. Segmental motion of all myocardial points can easily be quantitated and visualized from the location, direction, and length of the displayed vectors. An akinetic area in the lowerright area is indicated. 


\section{Myocardial Strains}

Starting at any time point during the cardiac cycle, the heart's motion is viewed here as a mapping which warps the deformed tag configuration into the undeformed configuration: $\mathbf{X}=\boldsymbol{\Gamma}(\mathbf{x})$. Equivalently, with $\mathbf{X}=\boldsymbol{V}(\mathbf{x})+\mathbf{x}$, the deformation gradient tensor can be written as $\mathbf{F}=\nabla \boldsymbol{\Gamma}(\mathbf{x})=\nabla \boldsymbol{V}(\mathbf{x})+\nabla \mathbf{x}$ where $\boldsymbol{V}$ is the computed displacement vector field. Therefore, assuming little or no throughplane motion, the strain tensor can be computed from spatial derivatives of displacements:

$$
\begin{aligned}
\mathbf{E} & =\frac{1}{2}\left(\mathbf{F}^{T} \mathbf{F}-\mathbf{I}\right) \\
& =\left(\begin{array}{cc}
u_{x}+\frac{1}{2}\left(u_{x}^{2}+v_{x}^{2}\right) & \frac{1}{2}\left(u_{y}+v_{x}+u_{x} u_{y}+v_{x} v_{y}\right) \\
\frac{1}{2}\left(u_{y}+v_{x}+u_{x} u_{y}+v_{x} v_{y}\right) & v_{y}+\frac{1}{2}\left(u_{y}^{2}+v_{y}^{2}\right)
\end{array}\right)
\end{aligned}
$$

Once a displacement vector field is available, the strain of deformation can be computed at all myocardial points within a short-axis slice. Furthermore, the quantity $\mathbf{M}^{T} \mathbf{E M}$ will give the value of strain for the direction $\mathbf{M}$.

\section{In-Vivo Imaging}

A SPAMM pulse sequence was used to collect images from a porcine model of myocardial infarction. The repetition time (TR) of the imaging sequence was approximately $7.1 \mathrm{msec}$, the echo time (TE) was $2.9 \mathrm{msec}$, and the rf pulse flip angle was 15 degrees. Other imaging parameters were: field of view $=300 \mathrm{~mm}$, data acquisition matrix size $=250 \times 256$ (phase encoding by readout), in-plane resolution $=1.2 \times 1.17 \mathrm{~mm}^{2}$, slice thickness $=7 \mathrm{~mm}$, and tag spacing $=7 \mathrm{~mm}$.

\subsection{Results}

Figure 4 shows 4 displacement vector fields ( V) computed from grids in tagged short-axis images at 202, 225, 247, and $270 \mathrm{msec}$ after the ECG trigger in a pig after inducing an anterior MI. The magnitude of the motion fields for each of these figures can easily be computed. Although the infarct zone can readily be recognized from the vector fields, these zones more clearly stand out in the vector field magnitude maps [5]. The next pair of figures: figure 5 and figure 6 show the radial and circumferential strains for the post-MI study after application of a median filter for reducing noise. Finally, figure 7 shows the picture of a stained histological slice of the LV roughly corresponding to the position of image slices for the porcine model.

In summary, the areas of the myocardium which lack motion can be recognized from either the magnitude of the displacement vectors or from the patterns of radial and circumferential strain maps. These areas appear to correlate well with the stained tissue. 

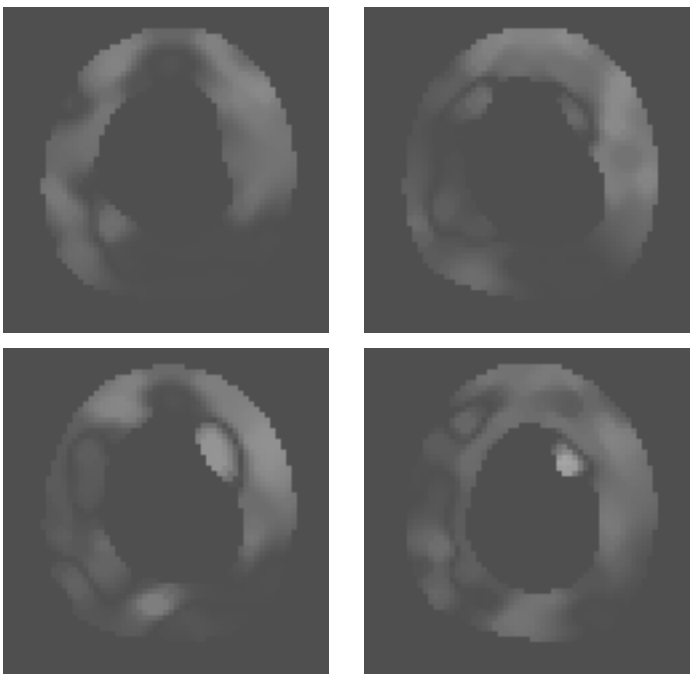

$-0.32$

Fig. 5. This figure shows radial strains for the post MI study (corresponding to the same time points as in figure 4). Notice that the lower-right area consistently has small strain values.

\section{Conclusions}

We have compared land-mark based and curve-based thin-plate warps for reconstruction of dense displacement vector fields from SPAMM grids. Using the Cardiac Motion Simulator, it was found that for larger deformations, land-mark based warps fail when few homologous land-mark points are available, but that performance gracefully diminishes for curve-based warps when the number of homologous curves are decreased. In addition, preliminary results from application of methods to in-vivo data of a porcine model of myocardial infarction were given. Although we have not taken into account motion in the out-of-plane direction, we are encouraged by the in-plane results obtained thusfar.

\section{Acknowledgements}

This work was supported in part by a grant from the Whitaker Biomedical Engineering Foundation, grant IRI-9796207 from the NSF, and grant HL-57628 from the NIH. 

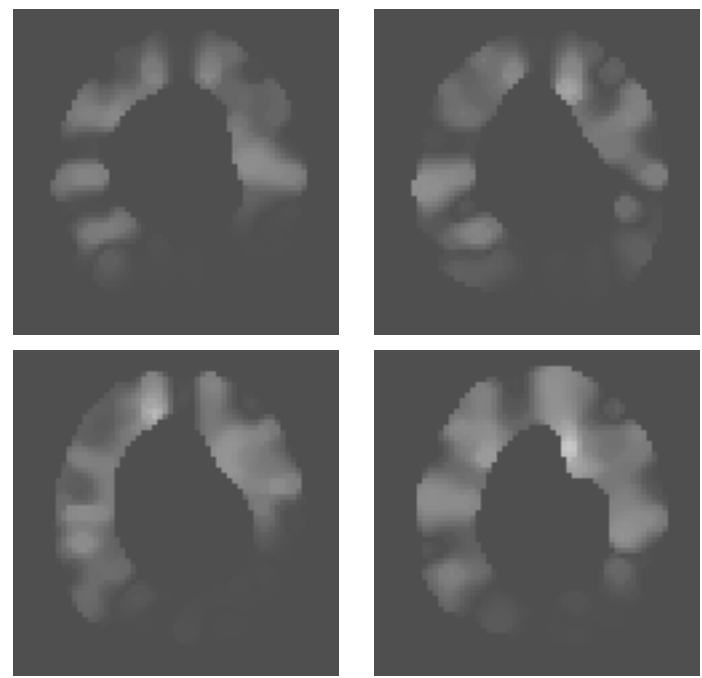

$-0.31$

Fig. 6. This figure shows circumferential strains for the post MI study (same time points as in figure 4). Once again, the lower-right area consistently has small strain values.

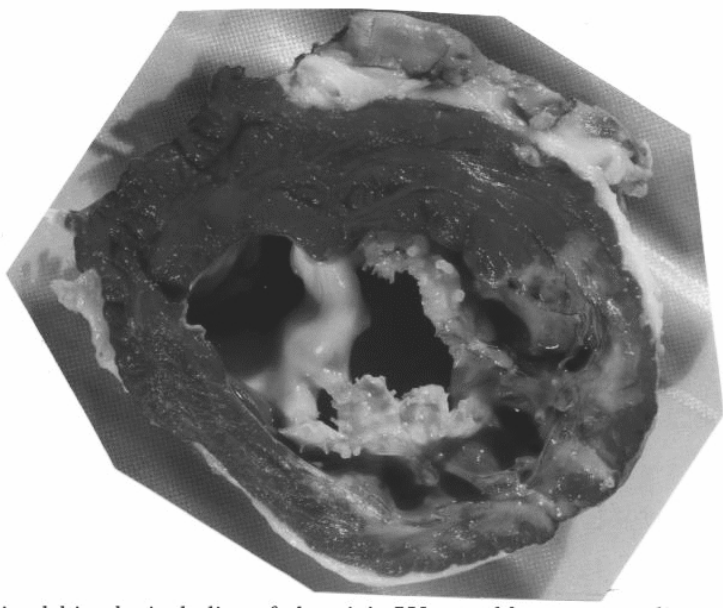

Fig. 7. Stained histological slice of the pig's LV; roughly corresponding to collected short-axis image slice positions. The area in the lower right (between 2 and 7 o'clock locations) of the picture with no dye uptake (bright region) is the infarct zone. Note also the distinct narrowing of the myocardial regions in these areas. 


\section{References}

1. A. A. Amini. Automated techniques for measurement of cardiac motion from MR tagging. Proposal funded by The Whitaker Foundation, 1992. 499

2. A. A. Amini and et al. MR physics-based snake tracking and dense deformations from tagged MR cardiac images. In AAAI Symposium on Applications of Computer Vision to Medical Image Processing, Stanford University, Stanford, California, March 1994. 499

3. A. A. Amini, R. W. Curwen, and J. C. Gore. Snakes and splines for tracking nonrigid heart motion. In European Conference on Computer Vision, pages 251-261, University of Cambridge, UK, April 1996. 499

4. A. A. Amini, Y. Chen, R. Curwen, V. Mani, and J. Sun. Coupled B-Snake Grids and Constrained Thin-Plate Splines for Analysis of 2D Tissue Deformations from Tagged MRI. IEEE Trans. on Medical Imaging, 17(3):344-356. 499, 500, 501

5. A. A. Amini, Y. Chen, D. Abendschein. Comparison of Land-Mark-Based and Curve-Based Thin-Plate Warps for Analysis of Left-Ventricular Motion from Tagged MRI. CVIA Lab Internal Report. March 1999. 504

6. T. Arts, W. Hunter, A. Douglas, A. Muijtjens, and R. Reneman. Description of the deformation of the left ventricle by a kinematic model. J. Biomechanics, 25(10):1119-1127, 1992. 501

7. L. Axel and L. Dougherty. MR imaging of motion with spatial modulation of magnetization. Radiology, 171(3):841-845, 1989. 498

8. F. Bookstein. Principal warps: Thin-plate splines and the decomposition of deformations. IEEE Transactions on Pattern Analysis and Machine Intelligence, PAMI-11:567-585, 1989. 499

9. W. Grossman. Assessment of regional myocardial function. J. of Amer. Coll. of Cardiology, 7(2):327 - 328, 1986. 498

10. S. Gupta and J. Prince. On variable brightness optical flow for tagged MRI. In Information Processing in Medical Imaging (IPMI), pages 323-334, 1995. 498

11. J. Park, D. Metaxas, and L. Axel. Volumetric deformable models with parameter functions: A new approach to the $3 \mathrm{~d}$ motion analysis of the LV from MRI-SPAMM. In International Conference on Computer Vision, pages 700-705, 1995. 498

12. P. Radeva, A. Amini, and J. Huang. Deformable B-Solids and implicit snakes for 3d localization and tracking of SPAMM MRI data. Computer Vision and Image Understanding, 66(2):163-178, May 1997.

13. N. Reichek. Magnetic resonance imaging for assessment of myocardial function. Magnetic Resonance Quarterly, 7(4):255-274, 1991. 499

14. D. Terzopoulos. Multiresolution Computation of Visible Representation. PhD thesis, MIT, 1984. 500

15. E. Waks, J. Prince, and A. Douglas. Cardiac motion simulator for tagged MRI. In Proc. of Mathematical Methods in Biomedical Image Analysis, pages 182-191, 1996. 501

16. A. Young, D. Kraitchman, L. Dougherty, and L. Axel. Tracking and finite element analysis of stripe deformation in magnetic resonance tagging. IEEE Transactions on Medical Imaging, 14(3):413-421, September 1995. 498

17. E. Zerhouni, D. Parish, W. Rogers, A. Yang, and E. Shapiro. Human heart: Tagging with MR imaging - a method for noninvasive assessment of myocardial motion. Radiology, 169:59-63, 1988. 498 